\title{
DSpace@MIT
}

MIT Open Access Articles

\author{
Electronic Spin Transition in Nano- \\ size Stoichiometric Lithium Cobalt Oxide
}

The MIT Faculty has made this article openly available. Please share how this access benefits you. Your story matters.

Citation: Qian, Danna, Yoyo Hinuma, Hailong Chen, Lin-Shu Du, Kyler J. Carroll, Gerbrand Ceder, Clare P. Grey, and Ying S. Meng. “Electronic Spin Transition in Nanosize Stoichiometric Lithium Cobalt Oxide." Journal of the American Chemical Society 134, no. 14 (April 11, 2012): 6096-6099.

As Published: http://dx.doi.org/10.1021/ja300868e

Publisher: American Chemical Society (ACS)

Persistent URL: http://hdl.handle.net/1721.1/80305

Version: Author's final manuscript: final author's manuscript post peer review, without publisher's formatting or copy editing

Terms of Use: Article is made available in accordance with the publisher's policy and may be subject to US copyright law. Please refer to the publisher's site for terms of use. 


\section{Electronic Spin Transition in Nano-size Stoichiometric Lithium Cobalt Oxide}

\begin{tabular}{|r|l|}
\hline Journal: & Journal of the American Chemical Society \\
\hline Manuscript ID: & ja-2012-00868e.R1 \\
\hline Manuscript Type: & Communication \\
\hline Author: & nubmitted by the \\
\hline Complete List of Authors: & $\begin{array}{l}\text { Qian, Danna; University of California San Diego, } \\
\text { Hinuma, Yoyo; Kyoto University, Department of Materials Science } \\
\text { and Engineering } \\
\text { Chen, Hailong; Massachusetts Institute of Technology, Materials } \\
\text { Sciences and Engineering } \\
\text { Du, Lin-Shu; University of New York at Stony Brook, Department of } \\
\text { Chemistry } \\
\text { Carroll, Kyler; University of California, San Diego, Department of } \\
\text { Nano Engineering } \\
\text { Ceder, Gerbrand; Massachusetts Institute of Technology, Dept. of } \\
\text { Materials Science \& Engineering } \\
\text { Grey, Clare; University of Cambridge, Chemistry } \\
\text { Meng, Ying; University of California San Diego, Department of Nano } \\
\text { Engineering }\end{array}$ \\
\hline
\end{tabular}

\section{SCHOLARONE $^{\text {tw }}$ Manuscripts}


Lithium cobalt oxide $\left(\mathrm{LiCoO}_{2}\right)$ is a compound of great importance, as it has been the most widely used positive electrode material for lithium ion batteries for nearly two decades. Li$\mathrm{CoO}_{2}$ adopts the $\alpha-\mathrm{NaFeO}_{2}$-type crystal structure with rhombohedral symmetry (space group $\mathrm{R}-3 \mathrm{~m}$ ) and $\mathrm{Li}^{+}$and $\mathrm{Co}^{3+}$ ions sitting in octahedral sites formed by alternating layers of oxygen. Because of such an ordered layered structure, $\mathrm{Li}^{+}$can be reversibly deintercalated and re-intercalated from $\mathrm{LiCoO}_{2}$ to $\mathrm{Li}_{0.5} \mathrm{CoO}_{2}$ with a high electrochemical potential of up to $4.2 \mathrm{~V}$ (vs $\mathrm{Li}^{+} / \mathrm{Li}$ ). In recent years, it has been demonstrated that ultra fast charge/discharge rate capabilities can be achieved in this compound when nano-scale $(<50 \mathrm{~nm})$ particles with morphology optimal for $\mathrm{Li}$ intercalation are prepared and tested. ${ }^{1,2}$ Okubo et al. ${ }^{1,3}$ observed several interesting phenomena associated with their nano-sized $\mathrm{LiCoO}_{2}$ : First, lattice parameter expansion is observed in particles less than $20 \mathrm{~nm}$; second, the magnetic susceptibility increases dramatically when compared with that of bulk- $\mathrm{LiCoO}_{2}$. The authors hypothesized that these phenomena are mainly due to the presence of $\mathrm{Co}^{2+}$ on the surface of their nano-sized particles, $\mathrm{Co}^{2+}$ being present in the form of $\mathrm{Li}_{1+\mathrm{x}} \mathrm{Co}^{2+}{ }_{\mathrm{x}} \mathrm{Co}^{3+}{ }_{1-\mathrm{x}} \mathrm{O}_{2}$, as a consequence of their hydrothermal synthetic process. However, the interpretation of the magnetic data is ambiguous since no direct evidence for the presence of $\mathrm{Co}^{2+}$ was obtained. ${ }^{3}$ Moreover, Levasseur et al. ${ }^{4}$ pointed out that in bulk lithium over-stoichiometric ("Liexcess") samples, the charge is compensated by oxygen vacancies. This leads to some cobalt ions being in a square based pyramidal site with an intermediate spin (IS) configuration. In this communication we show that, for stoichiometric nanosized $\mathrm{LiCoO}_{2}$, the anomaly in magnetic susceptibility can similarly be explained by the presence of IS or $\mathrm{HS} \mathrm{Co}^{3+}$, a

\begin{abstract}
A change in the electronic spin state of the sur-
faces relevant to $\mathrm{Li}$ (de)intercalation of nano-sized stoichiometric lithium cobalt oxide $\mathrm{LiCo}(\mathrm{III}) \mathrm{O}_{2}$ from low-spin to intermediate and high spin is observed for the first time. (de)intercalation. From density functional theory calculations ered lithium cobalt oxide can be significantly lowered as a consequence of the spin change. The crystal field splitting of
$\mathrm{Cod}$ orbitals is modified at the surface due to missing Co-O bonds. The electronic spin transition also has a significant impact on $\mathrm{Co}(\mathrm{III})-\mathrm{Co}(\mathrm{IV})$ redox potential, as revealed by the
change in the lithium (de)intercalation voltage profile in a lithium half cell.
\end{abstract}

phenomenon which alters the lithium (de)intercalation voltage significantly.

The surface energies of $\mathrm{LiCoO}_{2}$ were calculated from first principles with the Hubbard $\mathrm{U}$ correction on the generalized gradient approximation $(\mathrm{GGA}+\mathrm{U})$ to the density functional theory (DFT). The results are listed in Table 1 and the specific details of the models are presented in the supporting information $\mathrm{S} 1$. We find that the surface energies are minimized when the surface $\mathrm{Co}^{3+}$ ions are either in the intermediate (IS) or high spin (HS) state depending on the crystallographic orientation. This change in the electronic spin state on the surface can be rationalized by a change in the surface Co crystal field due to the missing $\mathrm{Co}-\mathrm{O}$ bonds.

$\mathrm{LiCoO}_{2}$ particles are typically dominated by the $\{001\}$ surface, however this surface is not active for $\mathrm{Li}$ (de)intercalation. Therefore, we focus on studying the $\{104\}$ and $\{110\}$ surfaces, which are the two lowest energy non-polar surfaces identified by previous work. ${ }^{5}$ Figure 1a shows the $\{104\}$ surface of $\mathrm{LiCoO}_{2}$, which represents a major, low energy surface for $\mathrm{LiCoO}_{2}$; this surface slices through the $\mathrm{Co}, \mathrm{O}$ and Li planes and is expected to be involved in the (de)intercalation process. This represents the $\{100\}$ surface of the $\mathrm{NaCl}$ lattice from which the ordered rocksalt $\mathrm{LiCoO}_{2}$ is derived. In the bulk, and on the $\{001\}$ surface, octahedrally coordinated $\mathrm{Co}^{3+}$ ions are in the low spin (LS) state and do not have unpaired electrons. On the $\{104\}$ surface, however, the $\mathrm{Co}^{3+}$ ions are coordinated by five oxygen ions, resulting in a square pyramidal configuration. The surface energy is then lowered significantly when going from the LS configuration $\left(1118 \mathrm{~mJ} / \mathrm{m}^{2}\right)$ to the IS $\left(312 \mathrm{~mJ} / \mathrm{m}^{2}\right)$. Figure $1 \mathrm{~b}$ depicts the $\{110\}$ surface of $\mathrm{LiCoO}_{2}$. In this orientation, the $\mathrm{Co}^{3+}$ ions on the surface are coordinated by four oxygen ions with a pseudo-tetrahedral configuration. The surface energy is minimized from $2227 \mathrm{~mJ} / \mathrm{m}^{2}$ (LS) to $1241 \mathrm{~mJ} / \mathrm{m}^{2}$ (HS). The details of the crystal field splitting of $3 d$ orbitals and the corresponding energy levels of bulk and surface cobalt ions are shown in Figure 1c. A square pyramidal crystal field breaks the degeneracy of both the $t_{2 g}$ and $e_{g}$ orbitals observed for octahedral symmetry, since the missing $\mathrm{O}$ ion along the $\mathrm{z}$-direction reduces the repulsion between $2 p$ electrons and $3 d$ electrons in orbitals pointing towards or closer to the z-axis. A pseudo-tetrahedral crystal field with two missing bonds in the $x y$ plane leads to lower energies for the $3 d_{x y}$ and $3 d_{x 2-y 2}$ orbitals. A charge density plot, which represents the difference in up and down spins, of the $\{104\}$ surface clearly show the unpaired electrons as compared to the case of $\mathrm{Co}^{3+}$ (LS) in the bulk of $\mathrm{LiCoO}_{2}$. (See Figure 2a and 2b) The first principles calculations reveal that both the $\{104\}$ and $\{110\}$ surfaces with optimized Co coordination environments and electronic states expand normal to the surface, the displace- 
ment being on the order of $0.1-0.2 \AA$. It is important to point out that such changes in electronic spin states are also seen in first principles simulations of surfaces of $\mathrm{CoO}$ and $\mathrm{Co}_{2} \mathrm{O}_{3}$, as well as $\mathrm{LiNiO}_{2}$.

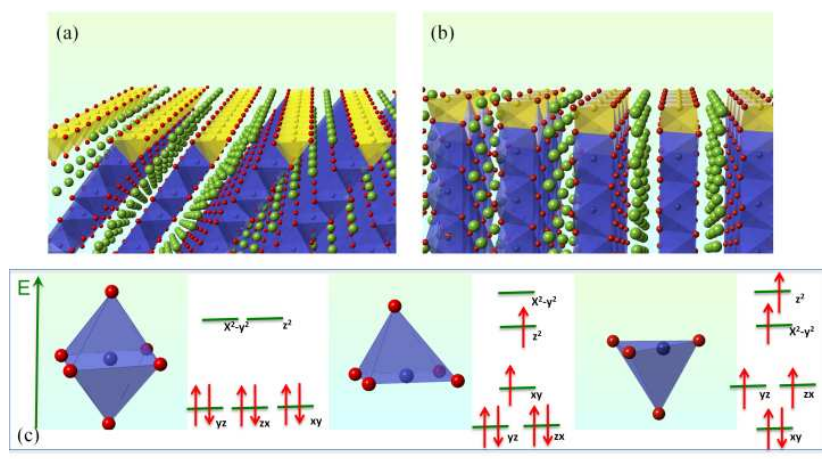

Figure 1. (a) $\{104\}$ and (b) $\{110\}$ surfaces of $\mathrm{LiCoO}_{2}$ (c) Octahedrally, square pyramid and pseudo-tetrahedrally coordinated Co ions. (red- oxygen, green - lithium, blue - Co)

To validate the hypothesis that the $\mathrm{Co}^{3+}$ on the surface is in an intermediate spin state by experimental spectroscopic techniques, stoichiometric $\mathrm{LiCoO}_{2}$ samples with extremely small particle sizes (thus very large surface areas) provide the best opportunities. However, it is difficult to make stoichiometric nanoparticles of $\mathrm{LiCoO}_{2}$ by conventional solid state or hydrothermal methods. ${ }^{6}$ Okubo et al. reported samples with small particle sizes ( 8 to $32 \mathrm{~nm}$ ), but the samples are most likely over-stoichiometric, i.e., $\mathrm{Li}_{1+\mathrm{x}} \mathrm{CoO}_{2}{ }^{1}$ By contrast, the molten salt method reported earlier ${ }^{2}$ represents a much better approach to prepare stoichiometric $\mathrm{LiCoO}_{2}$ nanoparticles. In this work, stoichiometric $\mathrm{LiCoO}_{2}$ with very small particle sizes $(10 \mathrm{~nm}, 16 \mathrm{~nm}, 20 \mathrm{~nm}, 30 \mathrm{~nm}$ and $40 \mathrm{~nm})$ were synthesized by using a modified molten salt method based on the previously reported method (see supporting information S2). We observed expansion of the lattice parameters from Rietveld analysis of the XRD results, similar to that observed by Okubo et al. ${ }^{1}$, along with magnetic susceptibility data that indicates the presence of unpaired electrons. X-ray diffraction (XRD) data indicates the formation of pure single-phase $\mathrm{LiCoO}_{2}$ for the 20, 30 and $40 \mathrm{~nm}$ samples, all the peaks corresponding to the layered $\alpha-\mathrm{NaFeO}_{2}$ structure (Figure 3a). Lattice parameters are extracted and the c/a ratio is approximately 4.99 , indicating a well-formed layered structure. ${ }^{7}$ Detailed information regarding the lattice parameters is given in the supporting information, S3. The reflections in the XRD pattern for the $10 \mathrm{~nm}$ particles are broad and a quantitative refinement could not be achieved.

X-ray photoelectron spectroscopy (XPS) was carried out with a Thermo-Scientific K-Alpha spectrometer using a focused monochromatic $\mathrm{Al} \mathrm{K \alpha}$ anode source (see supporting information S4). As shown in Figure 3b, all compounds show a Co $2 \mathrm{p}_{3 / 2}$ main peak at $779.5 \mathrm{eV}$ with a satellite peak at $789.5 \mathrm{eV}$ and a Co $2 \mathrm{p}_{1 / 2}$ main peak at $794.5 \mathrm{eV}$ with a satellite peak at $804.5 \mathrm{eV}$. This observation confirms that the oxidation state is $\mathrm{Co}^{3+}$. This is strong evidence that our nano-sized $\mathrm{LiCoO} 2$ samples are stoichiometric. $\mathrm{Co}^{2+}$ coordinated by oxygen is characterized by a strong broadening of the main line and a very intense satellite peak at $785.5 \mathrm{eV}\left(\mathrm{Co} 2 \mathrm{p}_{3 / 2}\right)$ and $802.5 \mathrm{eV}$ $\left(\mathrm{Co} 2 \mathrm{p}_{1 / 2}\right),{ }^{6,8}$ which are both absent in the spectra.
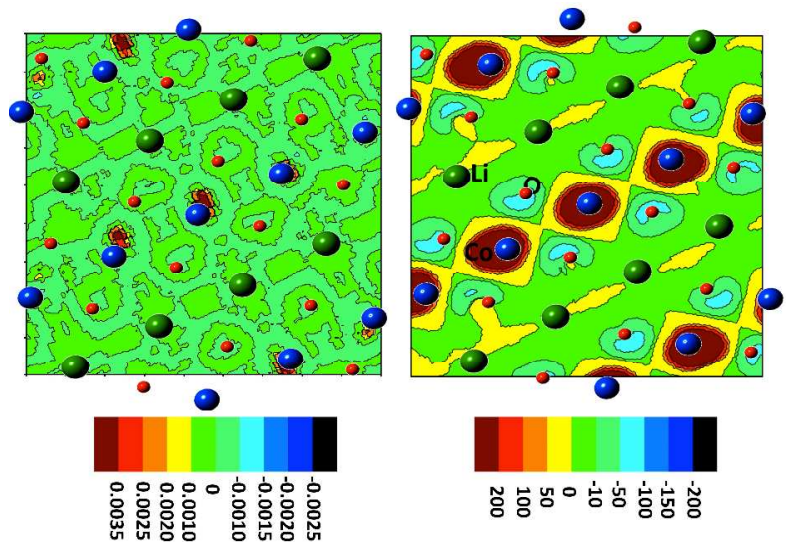

Figure 2. Spin density plot of the $\{104\}$ plane of the bulk (a) and the surface (b). (Notice the scale differences in the spin density)

${ }^{7}$ Li magic-angle-spinning (MAS) NMR spectroscopy was performed at a magnetic field strength of $4.7 \mathrm{~T}$ with a spinning speed of $35 \mathrm{kHz}$ to explore the Li nonstoichiometry in nanosized $\mathrm{LiCoO}_{2}$. Figure 4 a shows that the spectra of the 10 to 30 $\mathrm{nm}$ particles are dominated by a single resonance at $0 \mathrm{ppm}$, as expected for a stoichiometric, diamagnetic $\mathrm{LiCoO}_{2}$ sample. ${ }^{4}$ The larger NMR linewidth observed for the $10 \mathrm{~nm}$ sample compared to the others may be due to the presence of a trace amount $(<1 \%)$ of $\mathrm{Co}_{3} \mathrm{O}_{4}$ impurity, which results in faster transverse relaxation leading to larger line width. A weak, hyperfine shifted resonance is observed at $+185 \mathrm{ppm}$ for the $30 \mathrm{~nm}$ sample. This shift, along with peaks at 3, -6 -16, -40 ppm (not observed in our system here, presumably because they are too weak) were previously suggested to be associated with excess $\mathrm{Li}$ ions that replace $\mathrm{Co}^{3+}$ sites in the bulk, resulting in a defect structure discussed above, with two adjacent square-based pyramids containing two intermediate-spin $\mathrm{Co}^{3+}$ ions per oxygen vacancy. ${ }^{4}$ Even in the $30 \mathrm{~nm}$ sample, the percentage of excess $\mathrm{Li}$ is only approximately $0.6 \%$. Two new hyperfine-shifted resonances were observed at -115 and -260 ppm (Fig. 4b), which are attributed to the presence of intermediate and/or high spin $\mathrm{Co}^{3+}$ (IS, HS). To the best of our knowledge, these shifts have not been reported in the literature. An analysis of the spin density on the $\{104\}$ surface, as shown in Figure 2(b), indicates that the $\mathrm{Li}^{+}$ions on this surface contain negative spin density and will thus give rise to a negative shift. Integration of the spin density around the Li nucleus to $0.8 \AA$, using the approach developed by Carlier et al. ${ }^{9}$ confirms this observation and indicates that the Li nuclei in the subsequent $\{104\}$ surface is negative, while the spin density of the $\mathrm{Li}$ in the layer below is smaller, but positive (and thus it may be difficult to resolve from the intense bulk $\mathrm{LiCoO}_{2}$ resonance/and or be buried under the spinning sidebands). Further calculations will focus on the direct calculations of hyperfine shifts $^{10,11}$ on this and a wider range of surfaces. Importantly, quantitative fitting of the NMR spectra to extract the concentration of $\mathrm{Li}^{+}$ions nearby paramagnetic Co ions, confirms that the percentage of paramagnetic ions increases with decreasing particle size (Supporting information S5). 

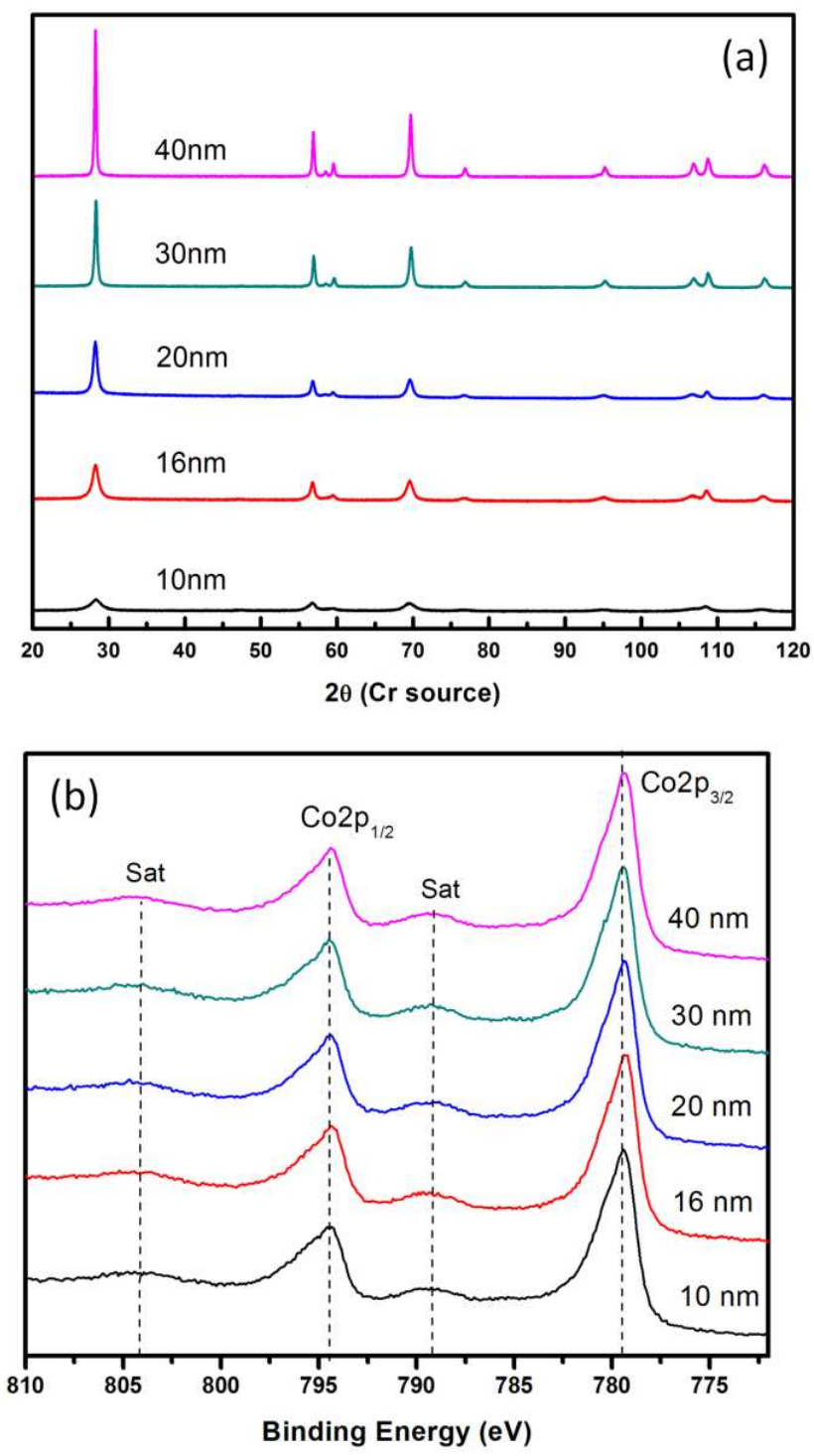

Figure 3. (a) XRD and (b) XPS spectra of nano-sized stoichiometric $\mathrm{LiCoO}_{2}$.

Magnetic measurements were performed, using a superconducting quantum interference device (SQUID), at a magnetic field of $1.0 \mathrm{~T}$ in the temperature range of $5-300 \mathrm{~K}$. The molar magnetic susceptibility of the various sized nano- $\mathrm{LiCoO}_{2}$ particles are plotted as a function of temperature in Figure 5a. The magnetic susceptibility of bulk $\mathrm{LiCoO}_{2}$ is low and practically temperature independent, which is attributed to a Van Vlecktype of paramagnetism associated with diamagnetic $\mathrm{Co}^{3+}(\mathrm{LS})$ in the layered structure. ${ }^{12}$ In contrast, our nano-size $\mathrm{LiCoO}_{2}$ exhibits a typical Curie-Weiss behavior for $\mathrm{T}>100 \mathrm{~K}$. The $\mathrm{Cu}-$ rie constant was determined in the temperature range of 200$300 \mathrm{~K}$. The Curie constant increases with decreasing particle size and fitting of the Curie constant indicates that the molar Curie constant is as high as 0.20 in $10 \mathrm{~nm}$-sized $\mathrm{LiCoO}_{2}$. (Details on the Curie constant fitting can be found in the supporting information S6).

Careful transmission electron microscopy (TEM) examination of the nano-sized $\mathrm{LiCoO}_{2}$ shows that all particles exhibit platelike morphology, plates corresponding to the $\{001\}$ planes; the edges are dominated by the $\{104\}$ planes justifying our computational study of this surface. (See TEM images in the sup- porting information S7). If the planes are terminated by the $\{001\}$ surface, according to first principles computation of this work and previous work by Kramer and Ceder, ${ }^{5} \mathrm{Co}^{3+}$ remains octahedrally coordinated with a low-spin configuration. The contribution of $\mathrm{Co}^{3+}$ (IS) on the $\{104\}$ and the $\mathrm{Co}^{3+}$ (HS) on the $\{110\}$ surfaces are most likely attributing to the abnormally high magnetic susceptibility seen in nano-size $\mathrm{LiCoO}_{2}$.

The proposed electronic spin state of the surfaces relevant for (de)intercalation has significant impact on the lithium (de)intercalation voltage profile, as revealed by first principles computation performed to calculate the Li extraction potential from different surfaces. If $\mathrm{Co}^{3+}$ remains as LS on the $\{104\}$ surface, the voltage of lithium extraction is as low as $2.32 \mathrm{~V}$; by contrast, when a spin transition from LS to IS occurs, the voltage of lithium extraction is $3.69 \mathrm{~V}$, close to the bulk value of $3.65 \mathrm{~V}$. The narrowing energy gap between the occupied and unoccupied states in IS and HS state Co ions indicates that the insulating nature of $\mathrm{LiCoO}_{2}$ can be altered due to the change in spin state. (See density of state plots in supporting information S8) This helps in explaining the fact that in the lithium half-cell with nano-sized $\mathrm{LiCoO}_{2}$ as the cathode, a clear first order phase transition, associated with the metalinsulator-transition, is absent as no flat voltage plateau is observed upon the first charge ${ }^{15}$ (also see supporting information S9).

(a)

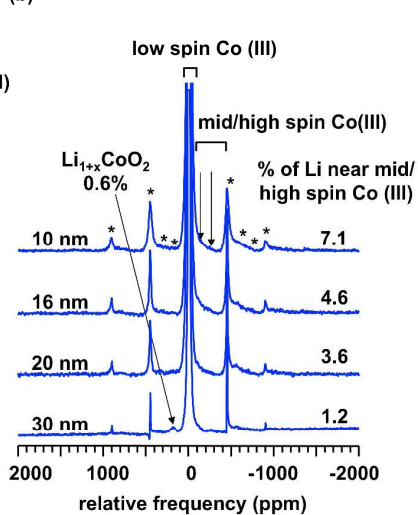

Figure 4. ${ }^{7} \mathrm{Li}$ MAS NMR spectra of $\mathrm{LiCoO}_{2}$ with varying particle sizes. (a) Percentages of the Li near by low spin $\mathrm{Co}$ (III) in $\mathrm{Li}$ -

$\mathrm{CoO}_{2}$ are listed in (a). Peak assignment and percentages of $\mathrm{Li}$ ions in environments nearby paramagnetic ions can be found in enlarged spectra. (b) Asterisks denote spinning sidebands.

In conclusion, it is proposed in this work that electronic spin state transitions occur on the surfaces of stoichiometric Li$\mathrm{CoO}_{2}$, where trivalent cobalt ions adjacent to the surface adopt an intermediate spin state if it they are square pyramid coordinated and a high spin state if they are pseudo-tetrahedrally coordinated. This phenomenon is quantified in nano-sized stoichiometric $\mathrm{LiCoO}_{2}$. We also observed in first principles calculations that both $\{104\}$ and $\{110\}$ surfaces with optimized electronic spin states expand normal to the surface and the displacement is of the order of 0.1-0.2 $\mathrm{A}$. This work suggests that changes in electronic spin state could be a common phenomenon in transition metal oxides. The low coordinated geometries on the surface of the oxides result in spin states that are distinct from the bulk. Consequently, unique magnetic and electronic properties arise and alter the materials performance in devices. We show that in this case, the voltage profile 
of (de)intercalation is dramatically changed. It is therefore promising to control the surfaces and interfaces of nano-sized materials to alter the electronic and magnetic properties, significantly different from the bulk behaviors.
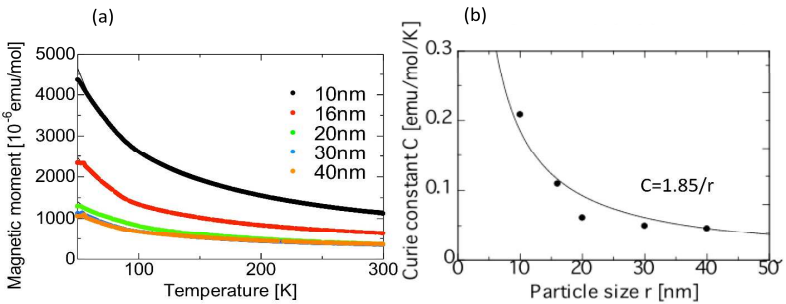

Figure 5. (a) Molar magnetic susceptibilities of particle size $10 \mathrm{~nm}, 16 \mathrm{~nm}, 20 \mathrm{~nm}, 30 \mathrm{~nm}$ and $40 \mathrm{~nm}$ samples as a function of temperature. (b) The Curie constants of these samples determined from the $1 / \chi$ vs T. (Both are measured with sample holder corrected)

TABLE 1.

\begin{tabular}{lllll}
\hline Surface & $\begin{array}{l}\text { Coordination } \\
\text { \# of oxygen }\end{array}$ & $\begin{array}{l}\gamma\left(\mathrm{mJ} / \mathrm{m}^{2}\right) \\
\text { ref }^{5} \\
\text { with LS }\end{array}$ & $\begin{array}{l}\gamma\left(\mathrm{mJ} / \mathrm{m}^{2}\right) \\
\text { This work } \\
\text { with LS }\end{array}$ & $\begin{array}{l}\gamma\left(\mathrm{mJ} / \mathrm{m}^{2}\right) \\
\text { this work } \\
\text { spin trans. }\end{array}$ \\
\hline$\{104\}$ & $5 / 6$ & 1048 & 1118 & 312 \\
$\{110\}$ & $4 / 6$ & 2241 & 2227 & 1241 \\
\hline
\end{tabular}

\section{ASSOCIATED CONTENT}

Supporting Information: Computation and experiment details, fitting for Curie Constant, morphology, electrochemical measurement, density of states (DOS) plots. This material is available free of charge via the Internet at http://pubs.acs.org.

\section{AUTHOR INFORMATION}

\section{Corresponding Author}

* Email: shirleymeng@ucsd.edu

\section{Author Contributions}

The manuscript was written through contributions of all authors. / All authors have given approval to the final version of the manuscript.

\section{Funding Sources}

The authors acknowledge financial support from the Northeastern Center for Chemical Energy Storage (NECCES), an Energy Frontier Research Center funded by the U.S. Department of Energy, Office of Science, Office of Basic Energy Sciences under Award Number DE-SC 0001294.

\section{ACKNOWLEDGMENT}

XPS is carried out at the ORNL Shared Research Equipment (SHaRE) User Facility, which is sponsored by the Office of Basic Energy Sciences, U.S. Department of Energy. Magnetic susceptibility measurement is done at Dr. Eric Fullerton's facility at UCSD. We thank the assistance from Dae-Hoe Lee and helpful discussions with Dr. Derek Middlemiss.

\section{ABBREVIATIONS}

XRD, XPS, SQUID, MAS-NMR, TEM

\section{REFERENCES}

(1) Okubo, M.; Hosono, E.; Kim, J.; Enomoto, M.; Kojima, N.; Kudo, T.; Zhou, H. S.; Honma, I. J. Am. Chem. Soc. 2007, 129, 7444.

(2) Chen, H.; Grey, C. P. Advanced Materials 2008, 20, 2206.

(3) Okubo, M.; Kim, J.; Kudo, T.; Zhou, H.; Honma, I. Journal of Physical Chemistry C 2009, 113, 15337.

(4) Levasseur, S.; Menetrier, M.; Shao-Horn, Y.; Gautier, L.; Audemer, A.; Demazeau, G.; Largeteau, A.; Delmas, C. Chemistry of Materials 2003, 15, 348 .

(5) Kramer, D.; Ceder, G. Chemistry of Materials 2009, 21, 3799.

(6) Daheron, L.; Dedryvere, R.; Martinez, H.; Menetrier, M.; Denage, C.; Delmas, C.; Gonbeau, D. Chemistry of Materials 2008, $20,583$.

(7) Ohzuku, T.; Makimura, Y. Chemistry Letters 2001, 642.

(8) Daheron, L.; Martinez, H.; Dedryvere, R.; Baraille, I.; Menetrier, M.; Denage, C.; Delmas, C.; Gonbeau, D. Journal of Physical Chemistry C 2009, 113, 5843.

(9) Carlier, D.; Ménétrier, M.; Grey, C. P.; Delmas, C.; Ceder, G. Physical Review B 2003, 67, 174103.

(10) Kim, J.; Middlemiss, D. S.; Chernova, N. A.; Zhu, B. Y. X.; Masquelier, C.; Grey, C. P. J. Am. Chem. Soc. 2010, 132, 16825. (11) Mali, G.; Meden, A.; Dominko, R. Chemical Communications 2010, 46, 3306.

(12) Kikkawa, S.; Miyazaki, S.; Koizumi, M. J. Solid State Chem. 1986, 62,35 .

(13) Buffat, B.; Demazeau, G.; Pouchard, M.; Hagenmuller, P. Mater Res Bull 1983, 18, 1153.

(14) Demazeau, G.; Pouchard, M.; Thomas, M.; Colombet, J. F.; Grenier, J. C.; Fournes, L.; Soubeyroux, J. L.; Hagenmuller, P. Mater Res Bull 1980, 15, 451.

(15) Chen, H.; Wu, L.; Zhang, L.; Zhu, Y.; Grey, C. P. J. Am. Chem. Soc., 2011, 133, 262.

Table of Contents artwork

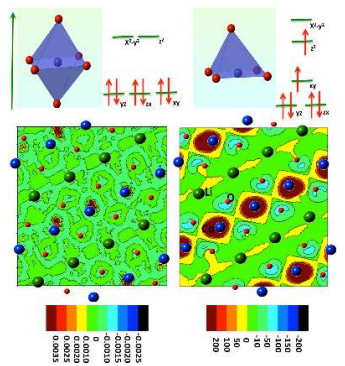

